OPEN ACCESS

Edited by:

André Canosa,

UMR6251 Institut de Physique de

Rennes (IPR), France

Reviewed by:

Maryvonne Gerin,

PSL research University, France

Marcelino Agundez,

Spanish National Research Council

(CSIC), Spain

*Correspondence:

C. Codella

claudio.codella@inaf.it

Specialty section:

This article was submitted to

Astrochemistry,

a section of the journal

Frontiers in Astronomy and Space

Sciences

Received: 23 September 2021 Accepted: 23 November 2021

Published: 23 December 2021

Citation:

Codella C, Ceccarelli C, Chandler C, Sakai N, Yamamoto S and The FAUST

Team (2021) Enlightening the

Chemistry of Infalling Envelopes and Accretion Disks Around Sun-Like Protostars: The ALMA FAUST Project. Front. Astron. Space Sci. 8:782006. doi: 10.3389/fspas.2021.782006

\section{Enlightening the Chemistry of Infalling Envelopes and Accretion Disks Around Sun-Like Protostars: The ALMA FAUST Project}

\author{
C. Codella ${ }^{1,2 *}$, C. Ceccarelli ${ }^{2}$, C. Chandler ${ }^{3}$, N. Sakai ${ }^{4}$, S. Yamamoto ${ }^{5}$ and The FAUST Team \\ ${ }^{1}$ INAF, Osservatorio Astrofisico di Arcetri, Firenze, Italy, ${ }^{2}$ Univ. Grenoble Alpes, CNRS, IPAG, Saint Martin d'Hères, France, \\ ${ }^{3}$ National Radio Astronomy Observatory, Socorro, NM, United States, ${ }^{4}$ RIKEN Cluster for Pioneering Research, Saitama, Japan, \\ ${ }^{5}$ Department of Physics, The University of Tokyo, Tokyo, Japan
}

The huge variety of planetary systems discovered in recent decades likely depends on the early history of their formation. In this contribution, we introduce the FAUST Large Program which focuses specifically on the early history of solar-like protostars and their chemical diversity at scales of $\sim 50 \mathrm{au}$, where planets are expected to form. In particular, the goal of the project is to reveal and quantify the variety of chemical composition of the envelope/ disk system at scales of 50 au in a sample of Class 0 and I protostars representative of the chemical diversity observed at larger scales. For each source, we propose a set of molecules able to (1) disentangle the components of the 50-2000 au envelope/disk system, (2) characterize the organic complexity in each of them, (3) probe their ionization structure, and (4) measure their molecular deuteration. The output will be a homogeneous database of thousands of images from different lines and species, i.e., an unprecedented source survey of the chemical diversity of solar-like protostars. FAUST will provide the community with a legacy dataset that will be a milestone for astrochemistry and star formation studies.

Keywords: astrochemistry, stars: formation, interstellar medium: molecules, interstellar medium: abundances, protostars

\section{INTRODUCTION: THE ROOTS OF FAUST}

Planets are a common product of the star formation process, and there is an incredible variety of planetary systems in the Galaxy (e.g., http://exoplanet.eu/), very different from the Solar System. The origin of such diversity, both in physics and chemistry, probably resides in the earliest history of the system formation, namely, what happens during the protostellar phases. The low-mass star formation is the complex process transforming a diffuse atomic cloud into, first, a dense molecular cloud and eventually into a Sun-like star surrounded by its planetary system. Meanwhile, the chemical composition of the gas involved in this process increases its complexity, from mostly atomic clouds to the so-called interstellar complex organic molecules (iCOMs), i.e., species with at least six atoms (e.g., methanol, $\mathrm{CH}_{3} \mathrm{OH}$ ), which can be considered as a brick to build pre-biotic chemistry (see e.g. Ceccarelli et al., 2007; Herbst and van Dishoeck, 2009; Caselli and Ceccarelli, 2012; Jørgensen et al., 2020, and references therein). Nowadays, evidence is mounting that the first steps of the process, namely, when the protostar is in the so-called Class $0\left(\sim 10^{4} \mathrm{yr}\right)$ and I ( $\left.\sim 10^{5} \mathrm{yr}\right)$ source phases (e.g., André et al., 2014, and references therein), are crucial for the future of the nascent planetary system. 


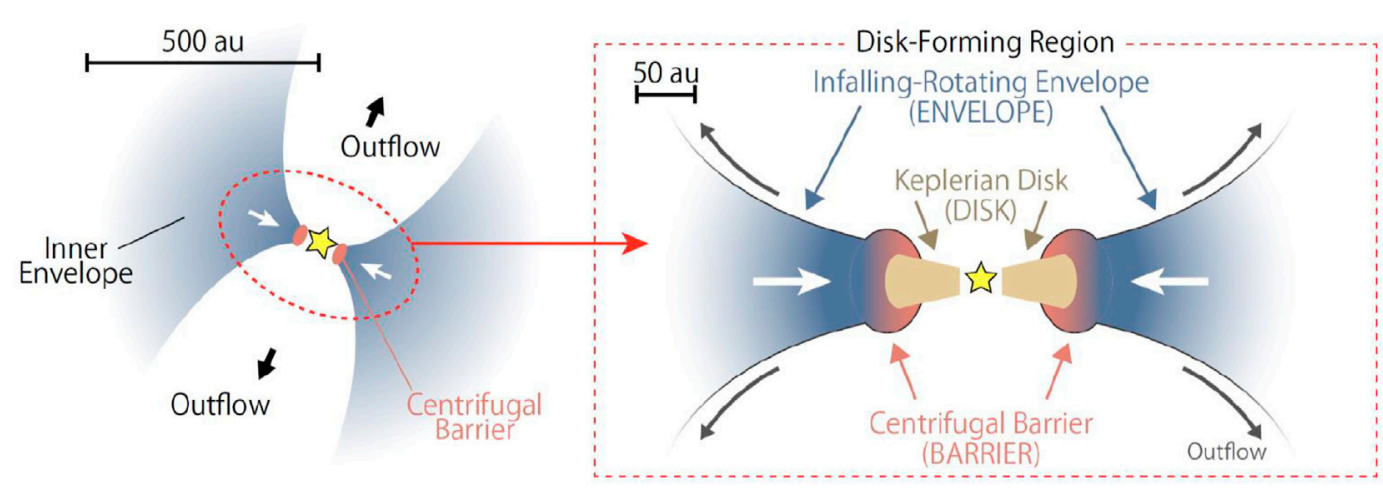

FIGURE 1 | Schematic structure of the region around solar-like Class 0 and I protostars, adapted from the study by Oya et al. (2016). Three main zones can be identified (see Section 2): the infalling and rotating envelope, the centrifugal barrier, and the rotating and accreting disk.

More specifically, a breakthrough result has been provided by the ALMA (Atacama Large Millimeter/submillimeter Array) interferometer, which provided images of rings and gaps (see e. g., Sheehan and Eisner, 2017; Andrews et al., 2018; Fedele et al., 2018) in the dust distribution around objects with an age less than 1 Myr. This supports that planet formation starts earlier than the classical protoplanetary stage (Class II; $\sim 10^{6} \mathrm{yr}$ ). These findings then support the importance of investigating the chemical complexity associated with protostars younger than $1 \mathrm{Myr}$.

In a nutshell, a Sun-like protostar is accreting its mass through a disk rotating along the equatorial plane. The disk, expected with a radius less than $100 \mathrm{au}$, is perpendicular to fast $\left(\sim 100 \mathrm{~km} \mathrm{~s}^{-1}\right)$ jets removing the angular momentum excess (see e.g., Frank et al., 2014; Lee et al., 2017a). In turn, the disk is fed by a large-scale ( $\geq 1,000 \mathrm{au}$ ) rotating and infalling molecular envelope. The chemical composition of the envelopes surrounding solar-like Class 0 and I protostars on large scales (100-2000 au) can be very different. Two distinct classes have been discovered (1) the hot corinos (Ceccarelli et al., 2007) and the WCCC (warm carbon chain chemistry) sources (Sakai and Yamamoto, 2013). Hot corinos (as e.g., IRAS16293-2422) are compact $(\geq 100 \mathrm{au})$ and dense $\left(\geq 10^{7} \mathrm{~cm}^{-3}\right)$ regions, where the temperature is warm enough ( $\geq 100 \mathrm{~K}$ ) to thermally evaporate the frozen dust mantles. As a consequence, the gas phase is chemically enriched in iCOMs due to either direct release from dust mantles or formed in the gas phase using simpler molecules from the mantles. On the other hand, WCCC sources (as e.g., L1527) are deprived of such iCOMs and enriched with unsaturated carbon-chain species, such as $\mathrm{HC}_{2 n+1} \mathrm{~N}, \mathrm{C}_{\mathrm{n}} \mathrm{H}$, and $\mathrm{C}_{\mathrm{n}} \mathrm{H}_{2}$ (Higuchi et al., 2018, and references therein).

In this context, breakthrough open questions are (i) whether such diversity is also present in the inner envelope/ disk system ( $\sim 50 \mathrm{au})$ and (ii) what molecules are passed from the large-scale envelope $(\sim 2000 \mathrm{au})$ to the disk in which planets, comets, and asteroids form. Yet, this is crucial to know because very likely the chemical composition and fate of the future planetary systems depend on the chemical class to which the original protostar belonged. The aim of this study is to introduce the ALMA (Atacama Large Millimeter/ submillimeter Array) Large Program (LP) FAUST (Fifty AU STudy of the chemistry in the disk/envelope system of Solar- like protostars; http://faust-alma.riken.jp), focused on astrochemistry of Class $0 / \mathrm{I}$ sources imaged at the Solar System spatial scale.

\section{THE FAUST PROJECT}

\subsection{Context and Main Goals}

The goal of FAUST is to characterize the gas chemical composition of the protostellar system, associated with several physical components at work to build a Sun-like star from a rotating and infalling envelope. FAUST builds up on previous surveys of the chemical composition of the Sun-like youngest protostars at larger spatial scales, such as ASAI (https://www.oan.es/asai/), and SOLIS (https://solis.osug.fr/), which covered the large envelopes at $\leq 5000$ au and the transition between the large-scale envelope and the circumstellar disk at $\geq 5000$ au and $\leq 100$ au, respectively. The FAUST approach is to identify three zones (see Figure 1), as learnt from previous ALMA studies (e.g., Sakai et al., 2014a, 2017):

- ENVELOPE: Here, we mean the infalling-rotating envelope on scales of a few 100 au. The gas chemical composition evolves from that at large ( $\sim 2000 \mathrm{au})$ scales because of the heating from the central object, which sublimates the grain mantles (Ceccarelli et al., 1996). Besides, the gas close to the outflow cavity wall might also be exposed to the UV and X-ray photons from the central object or be affected by mild transverse shocks (Stäuber et al., 2005), with a consequent chemical enrichment of the gas.

- BARRIER: The gas transits a centrifugal barrier, on scales of about $50 \mathrm{au}$, before entering the disk. The gas chemical composition may be drastically affected by the low velocity $\left(\sim 1 \mathrm{~km} \mathrm{~s}^{-1}\right)$ shock at the centrifugal barrier as grain mantles may be at least partially liberated into the gas phase and the gas heated and compressed (Sakai et al., 2014b,a; Oya et al., 2016). As a matter of fact, the IRAS $04386+2557$ protostar, in the L1527 Taurus core, can be considered the archetypal edge-on disk where Sakai et al. (2014b, 2017) revealed, for the first time, an increase of SO abundance at the radius of the centrifugal barrier. 
TABLE 1 | List of the frequency windows and of the species covered by the selected backends, divided in four groups (see Section 2.2).

\begin{tabular}{|c|c|c|c|c|c|}
\hline \multirow[t]{2}{*}{ Setup } & Frequency & \multirow[t]{2}{*}{ Zones } & Molecular & \multirow{2}{*}{$\frac{\text { Gas }}{\text { Ionization }}$} & \multirow{2}{*}{$\begin{array}{c}\text { Molecular } \\
\text { deuteration }\end{array}$} \\
\hline & (GHz) & & complexity & & \\
\hline 1 & $\begin{array}{l}214.0-219.0 \\
229.0-234.0\end{array}$ & $\begin{array}{l}\mathrm{C}-\mathrm{C}_{3} \mathrm{H}_{2}, \mathrm{CH}_{3} \mathrm{OH}, \mathrm{SO} \\
\mathrm{SiO}, \mathrm{H}_{2} \mathrm{CO}, \mathrm{C}^{18} \mathrm{O}\end{array}$ & $\begin{array}{l}\mathrm{CH}_{3} \mathrm{OH}, \mathrm{NH}_{2} \mathrm{CHO} \\
\mathrm{CH}_{3} \mathrm{CHO}, \mathrm{HCOOCH}\end{array}$ & $\mathrm{DCO}^{+}$ & $\mathrm{N}_{2} \mathrm{D}^{+}, \mathrm{D}_{2} \mathrm{CO}$ \\
\hline 2 & $\begin{array}{l}242.5-247.5 \\
257.5-262.5\end{array}$ & $\begin{array}{l}\mathrm{C}-\mathrm{C}_{3} \mathrm{H}_{2}, \mathrm{CS} \\
\mathrm{CH}_{3} \mathrm{OH}, \mathrm{SO}\end{array}$ & $\begin{array}{l}\mathrm{CH}_{3} \mathrm{OH}, \mathrm{NH}_{2} \mathrm{CHO} \\
\mathrm{CH}_{3} \mathrm{CHO}, \mathrm{HCOOCH} \\
\mathrm{CH}_{3} \mathrm{OCH}_{3}\end{array}$ & $\mathrm{H}^{13} \mathrm{CO}^{+}$ & $\mathrm{HDCO}, \mathrm{CH}_{2} \mathrm{DOH}$ \\
\hline 3 & $\begin{array}{c}85.0-89.0 \\
97.0-101.0\end{array}$ & $\mathrm{CH}_{3} \mathrm{OH}, \mathrm{HC}_{3} \mathrm{~N}$ & $\mathrm{CH}_{3} \mathrm{OH},{ }^{13} \mathrm{CH}_{3} \mathrm{OH}$ & $\mathrm{N}_{2} \mathrm{H}^{+}$ & $\mathrm{C}-\mathrm{C}_{3} \mathrm{HD}$ \\
\hline
\end{tabular}

Another enlightening case is represented by the HH212 pristine jet/disk system, located in Orion B. The disk, edge-on also in this case, shows iCOM-rich rotating rings, possibly associated with the centrifugal barrier with a radius of about 40 au (Lee et al., 2017b, 2019).

- DISK: At scales smaller than about $50 \mathrm{au}$, the gas settles in the rotationally supported disk, where the gas chemical composition is expected to be stratified (e.g., Aikawa and Herbst, 1999; Walsh et al., 2015) and affected by the dynamics and dust coagulation (e.g., Zhao et al., 2016; Tlee et al., 2017). Indeed, very recently, Podio et al., (2020) observed with ALMA the evolved Class I source IRAS $04302+2247$, where the bulk of the envelope has been dispersed; the molecular $\left(\mathrm{CO}, \mathrm{CS}\right.$, and $\left.\mathrm{H}_{2} \mathrm{CO}\right)$ emission is vertically stratified on a scale of 50-60 au (see also van't Hoff et al., 2020). A breakthrough result would be to reveal a chemical stratification in early protostellar disks still fully embedded in the envelope. Furthermore, the observations of protostellar disks are also challenged by the presence of jets and disk winds, which are powered at similar scales (e.g., Lee et al., 2017a; Tabone et al., 2017). For a proper study of disks, it is mandatory to reveal also the fast jet flowing perpendicular to the disk equatorial plane using e.g., a standard tracer as $\mathrm{SiO}$ (see e.g., Codella et al., 2019). Finally, at these small scales, dust opacity increases, which strongly affects the emission spectrum from the gas (see below).

Therefore, the three zones are expected to possess distinct physical and chemical properties, likely varying from source to source and/or depending on the star-forming regions. The goal of FAUST is to disentangle the three zones with the help of their kinematic signatures and ALMA line images at $50 \mathrm{au}$ spatial resolution. Our pioneering studies showing the proof of concept have already been conducted for a few sources and a few molecules (e.g., Ceccarelli et al., 2010; Oya et al., 2016; Codella et al., 2017): time is now ripe for a systematic study of many more sources and many more species, via an ALMA LP. In summary, the aim of FAUST is to reveal and quantify the variety of the chemical composition of the envelope/disk system of solar-like Class 0 and I protostars. Such chemical varieties will add a new dimension to the diversity of planetary systems, and we expect that it will have a substantial impact on studies of planet formation and the origin of the Solar System.

\subsection{Sources and Molecular Lines}

From a kinematical point of view, the envelope/disk system can be divided into three different zones. Although they could, in principle, be studied kinematically by observing $\mathrm{CO}$ and its isotopologue lines, but as a matter of fact, only rarer species provide a much more powerful diagnostic tool because they are differentially enhanced in the three zones, thanks to chemical composition changes (e.g., Sakai et al., 2014b, 2017; Oya et al., 2016). FAUST will simultaneously use kinematics and chemistry to fully resolve the complexity of the envelope/disk system. Four groups of species have been selected to probe different topics:

1. ZONE PROBES: $\mathrm{c}-\mathrm{C}_{3} \mathrm{H}_{2}, \mathrm{CS}, \mathrm{CH}_{3} \mathrm{OH}, \mathrm{SO}, \mathrm{SiO}, \mathrm{H}_{2} \mathrm{CO}, \mathrm{C}^{18} \mathrm{O}$, and $\mathrm{HC}_{3} \mathrm{~N}$. We select the following species to disentangle each zone: Envelope: $\mathrm{c}-\mathrm{C}_{3} \mathrm{H}_{2}$ and $\mathrm{CS}$. These species are present in the infalling-rotating envelope, with different abundances for different sources, but their abundance significantly drops in the other two zones so that they are specific in probing the ENVELOPE zone in different sources. Barrier: $\mathrm{CH}_{3} \mathrm{OH}$, SO, and $\mathrm{SiO}$. Their abundance is enhanced in the weak shocks at the centrifugal barrier. Narrow $\left(\sim 1 \mathrm{~km} \mathrm{~s}^{-1}\right) \mathrm{SiO}$ lines could trace the release of Si from dust mantles (Guillet et al., 2011; Lesaffre et al., 2013) at the centrifugal barrier, whereas broad ( $\geq 10 \mathrm{~km} \mathrm{~s}^{-1}$ ) $\mathrm{SiO}$ emission is expected to trace fast jets (associated with high-velocity shocks) perpendicular to the disk. Disk: $\mathrm{H}_{2} \mathrm{CO}, \mathrm{C}^{18} \mathrm{O}$, and $\mathrm{HC}_{3} \mathrm{~N}$. Given their relatively large abundance in the warm layers of the disks, lines from these species can probe the inner disk, via their high-velocity components.

2. MOLECULAR COMPLEXITY PROBES: $\mathrm{CH}_{3} \mathrm{OH}, \mathrm{NH}_{2} \mathrm{CHO}$, $\mathrm{CH}_{3} \mathrm{CHO}, \mathrm{CH}_{3} \mathrm{OCH}_{3}$, and $\mathrm{HCOOCH}_{3}$. A major goal of FAUST is measuring the organic complexity in the disk/ envelope system as it might be inherited at later stages by the nascent planetary system (Zhao et al., 2016). Based on previous studies, five species are particularly important to identify the organic diversity: methanol $\left(\mathrm{CH}_{3} \mathrm{OH}\right)$, formamide $\left(\mathrm{NH}_{2} \mathrm{CHO}\right)$, acetaldehyde $\left(\mathrm{CH}_{3} \mathrm{CHO}\right)$, dimethyl ether $\left(\mathrm{CH}_{3} \mathrm{OCH}_{3}\right)$, and methyl formate $\left(\mathrm{HCOOCH}_{3}\right)$. They are predicted to have a different chemical origin, with methanol a grain-surface product and formamide likely a gas-phase product (Codella et al., 2017; Skouteris et al., 

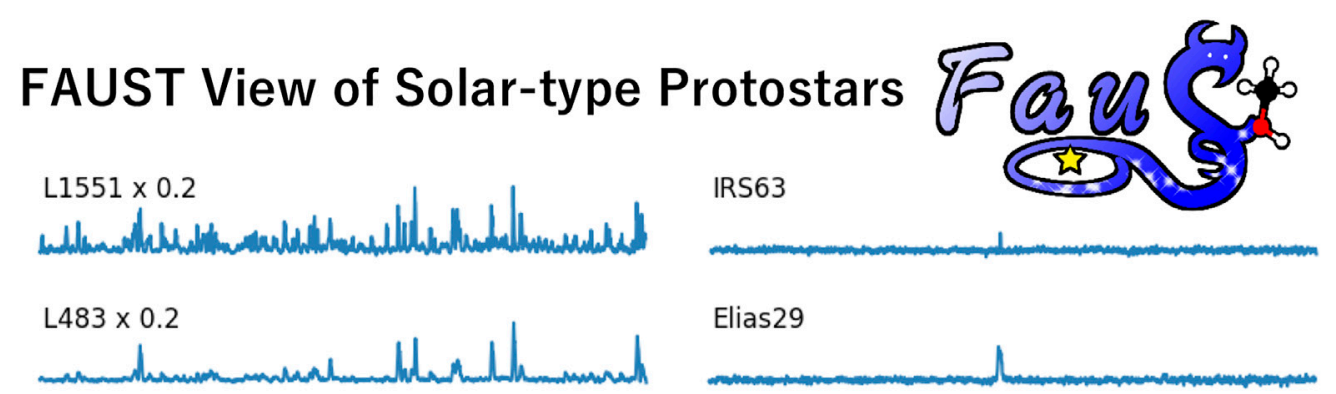

Elias29

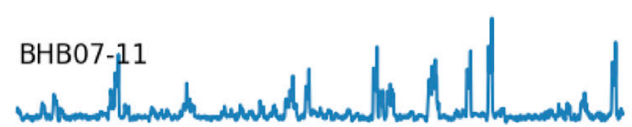

GSS30
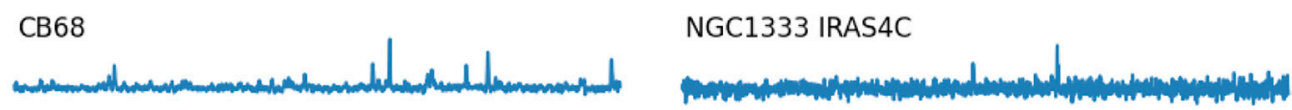

IRAS15398

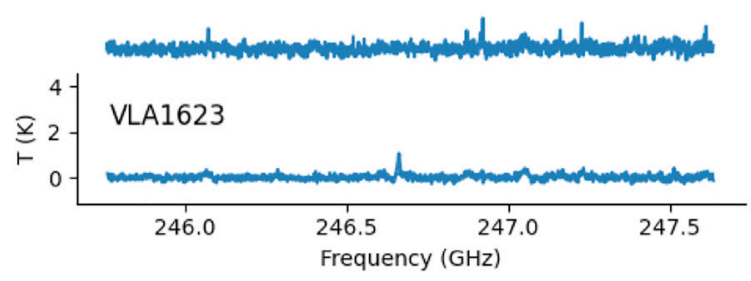

L1527

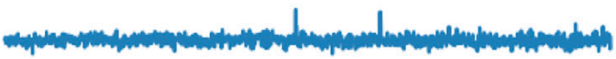

RCrA IRAS7B

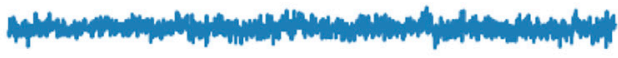

FIGURE 2 |Zoo of preliminary spectra observed towards FAUST sources (see black labels) in a portion of $2 \mathrm{GHz}(245.7-247.7 \mathrm{GHz}$ ) of the frequency Setup2 (See Table 1). The FAUST logo is also reported. The intensity scale is in brightness temperature (K). The spectra have been obtained merging ACA data with those at highspatial resolution. The identification of the lines is out of the focus of the present study. The goal of the sketch is to enlight the observed chemical differentiation, with some sources characterized by a rich spectra associated with iCOM emission.

2017), while for acetaldehyde, dimethyl ether, and methyl formate, the chemical synthesis route is largely debated (e.g., Garrod et al., 2008; Balucani et al., 2015).

3. GAS IONIZATION PROBES: $\mathrm{H}^{13} \mathrm{CO}^{+}, \mathrm{DCO}^{+}$, and $\mathrm{N}_{2} \mathrm{H}^{+}$. The degree of ionization in the inner 100 au envelope is a very important parameter for any theory of planet formation (e.g., Balbus and Hawley, 1998). Yet, this quantity is very poorly known at these scales. Several processes can affect it, from an inner source of energetic particles and/or X-rays (Stäuber et al., 2005) to the growth of dust grains (Zhao et al., 2016). Here, we propose to use molecular ions, following a previously used methodology, to estimate the gas ionization, such as the $\mathrm{H}^{13} \mathrm{CO}^{+} / \mathrm{DCO}^{+}$and $\mathrm{H}^{13} \mathrm{CO}^{+} / \mathrm{N}_{2} \mathrm{H}^{+}$abundance ratios. The first ratio measures the ionization in cold (smaller than $\sim 30 \mathrm{~K})$ and dense $\left(\geq 10^{4} \mathrm{~cm}^{-3}\right)$ gas (e.g., Caselli et al., 2008), while the second one in warm ( $\geq 40 \mathrm{~K}$ ) and, again, quite dense (up to $\sim 10^{7} \mathrm{~cm}^{-3}$ ) gas (e.g., Ceccarelli et al., 2014b).

4. DEUTERIUM-BEARING SPECIES: $\mathrm{c}-\mathrm{C}_{3} \mathrm{HD}, \mathrm{N}_{2} \mathrm{D}^{+}$, HDCO, $\mathrm{D}_{2} \mathrm{CO}$, and $\mathrm{CH}_{2} \mathrm{DOH}$ D-bearing species are powerful diagnostic tools to study the physical conditions at present and in the past (Ceccarelli et al., 2014a). Specifically, species like $\mathrm{c}-\mathrm{C}_{3} \mathrm{H}_{2}$ (one of the infalling-rotating envelope probe) and $\mathrm{N}_{2} \mathrm{D}^{+}$are present-day products, whereas $\mathrm{H}_{2} \mathrm{CO}$ and $\mathrm{CH}_{3} \mathrm{OH}$, being major components of the grain mantles, were mostly formed during the pre-stellar phase and introduced in the present-day gas by the presence of the accreting inner object. The respective deuterated counterparts provide, therefore, a precious tool to understand the physical conditions when these species are/were deuterated. In addition, molecular deuteration provides a sort of Ariadne's thread that links the ISM to the Solar System history (Ceccarelli et al., 2014a).

We selected a representative sample of sources that is known to exhibit a wide chemical composition diversity, based on the available large-scale observations. The following two criteria have been adopted: (1) Chemical diversity: Since, so far, the major chemical diversity is represented by WCCC and hot corino sources, we select sources that represent a continuous variation of the abundance ratio of species characteristics of these two classes. Specifically, (i) $\mathrm{CH}_{3} \mathrm{OH}$ can be regarded as a proxy of the hot corino species because it is a crucial organic molecule, considered a parent species of larger iCOMs; (ii) small hydrocarbons such as $\mathrm{C}_{2} \mathrm{H}$ or $\mathrm{c}_{-} \mathrm{C}_{3} \mathrm{H}_{2}$ are characteristics of WCCC sources. The selected sources are, therefore, associated with measured abundance ratios of these species varying by two orders of magnitude, covering the two extremes of hot corino and WCCC sources (Higuchi et al., 2018). (2) Distance and previously 


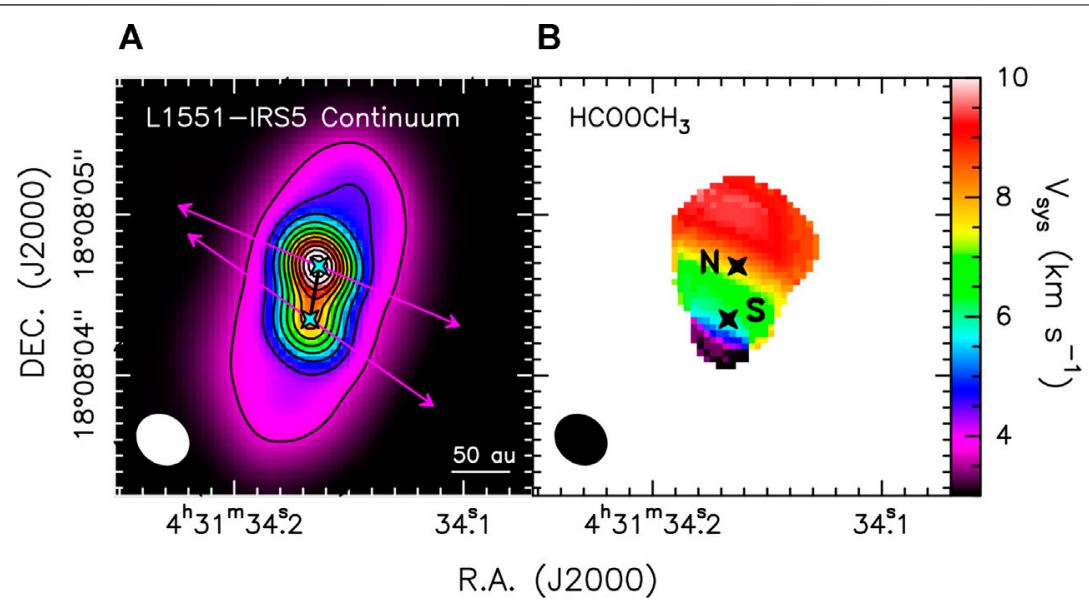

FIGURE 3 | L1551 IRS5 binary source, in Taurus. (A): $1.3 \mathrm{~mm}$ dust continuum emission in color scale and black contours (Bianchi et al., 2020): the N and S protostars are revealed. (B): Gas enriched in iCOMs around the IRS5 binary system, imaged on the 50 au scale. More specifically, the intensity-weighted velocity (also called moment 1) map of the $\mathrm{HCOOCH}_{3}\left(18_{4,14}-17_{4,13}\right) \mathrm{A}$ is shown, revealing a velocity gradient perpendicular to the axes of the protostellar jets (see the arrows in the $\left.\mathbf{( A )}\right)$ driven by $\mathrm{N}$ and $\mathrm{S}$. The systemic velocities are $+4.5 \mathrm{~km} \mathrm{~s}^{-1}$ (S object) and $+7.5 \mathrm{~km} \mathrm{~s}^{-1}(\mathrm{~N})$. The chemical enrichment occurs in rotating hot corinos around the protostars and/or in the rotating circumbinary disk.

\section{IRAS 15398-3359}

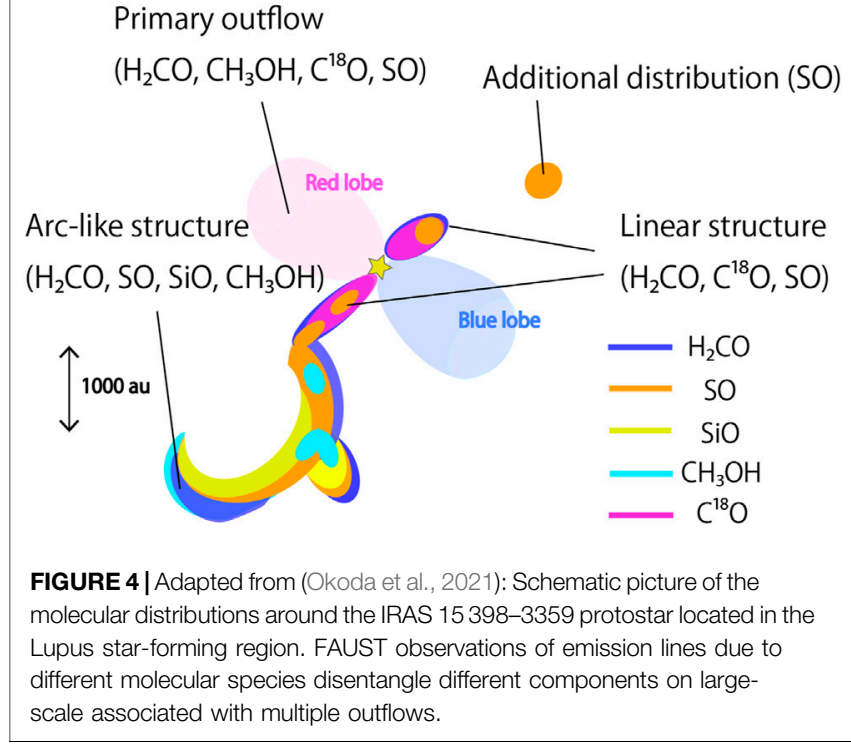

studied sources: We select nearby Class 0 and I sources with distance $\leq 250 \mathrm{pc}$ and a bolometric luminosity $\leq 25 \mathrm{~L} \square$.

The final list contains 13 sources located in different starforming regions: L1527 and L1551 IRS5 (the Taurus region), IRAS 15398-3359 (Lupus), L483 (Aquila), Elias 29, VLA1623A, IRS63, and GSS30 (Ophiucus), NGC1333 IRAS4A, and IRAS4C (Perseus), BHB07-11 (Pipe), RCrA IRS17A (Cr A), and CB68 (the isolated source). We will observe the three frequency settings in Table 1. They target lines from the four groups of molecules described above. We require a uniform linear resolution of $50 \mathrm{au}$ to identify the centrifugal barrier and to disentangle the disk from the envelope, namely, an angular resolution of 250-350 mas, depending on the distance of the source, and almost uniform brightness sensitivity. The high-angular scale of 50 au will be reached using the 12-m antenna ALMA array in different configurations. Furthermore, given the goal to also sample the molecular envelope, Atacama Compact Array (ACA/Morita Array) observations of 7-m antennas will be also performed. By combining all the visibility data obtained using both the $12-\mathrm{m}$ and the 7-m antenna array, FAUST will be able to follow the physical and chemical changes from the large-scale envelope $(\sim$ $2000 \mathrm{au}$ ) to the inner disk/jet system. For each frequency setting, we allocate one continuum setting to measure the dust spectral energy distribution (SED) from 3 to $1 \mathrm{~mm}$. The frequency resolution is set to $0.2 \mathrm{~km} \mathrm{~s}^{-1}$, i.e., the sound speed at $10 \mathrm{~K}$.

\subsection{Data Exploitation}

We will use a two-step modeling procedure to extract the information from the data, as follows: (1) Kinematics and radiative transfer models: The three zones are disentangled by comparing the velocity structure observed in the various molecular lines with kinematic models (Oya et al., 2016). The gas temperature and density structure, as well as the species column density, will be extracted from a multiline non-LTE (when possible) analysis using 1 and $3 \mathrm{D}$ codes (Bisbas et al., 2015; Zhao et al., 2016), taking into account the dust optical depth. The analysis of the continuum will provide the distribution of the $\mathrm{H}_{2}$ column density. (2) Astrochemical models: The derived molecular abundances will be compared with our astrochemical models (Ceccarelli et al., 2018; Wirström and Charnley, 2018), where the physical structure will be incorporated. From this analysis, we will extract valuable constraints on the reactions involved in the formation and destruction of the observed iCOMs. FAUST members with specific expertise will contribute to the project with laboratory experiments and 

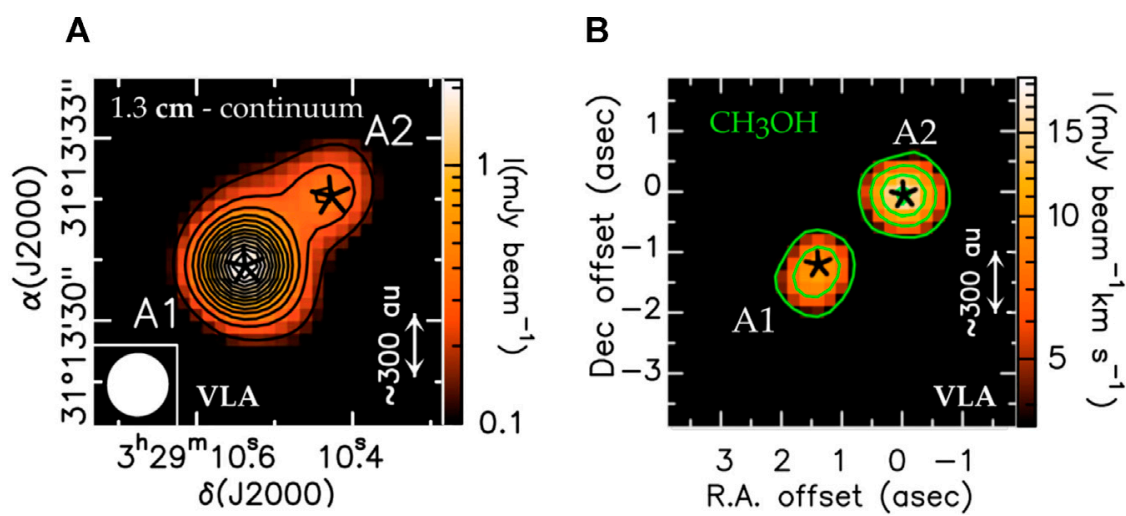

FIGURE 5 | (A): NGC1333 IRAS 4A continuum emission map at $25 \mathrm{GHz}$ as imaged using VLA (Adapted from De Simone et al. (2020)). Two protostars A1 and A2 are observed. (B): $\mathrm{CH}_{3} \mathrm{OH}\left(6_{2,4}-6_{1,5}\right)$ velocity-integrated maps in the color scale, revealing for the first time the hot-corino associated with $\mathrm{A} 1$, missed by sub-millimeter surveys due to dust opacity effects.

theoretical quantum chemistry calculations (Watanabe et al., 2010; Dulieu et al., 2013; Skouteris et al., 2017). In summary, we will determine (i) the extent to which the large-scale envelope diversity is conserved in the disk/envelope system and (ii) what molecules are passed from the large-scale envelope to the disk. In this way, the goal of the FAUST project will, therefore, be achieved.

\section{FIRST FAUST RESULTS AND PERSPECTIVES}

The analysis of the FAUST data is in progress. However, some instructive results have been already obtained. The data were reduced by using a modified version of the ALMA calibration pipeline and an additional in-house calibration routine (Okoda et al., 2021) to correct the system temperature and spectral line data normalization. Figure 2 reports an example of the zoo of FAUST spectra observed in a portion of the frequency Setup 2 (see Table 1) toward the protostellar coordinates. The spectra have been obtained merging ACA data with those at high-spatial resolution. The line identification and the discussion of the chemical content are out of focus of the present study; the goal of the spectra sketched in Figure $\mathbf{2}$ is to enlight a chemical differentiation, indicating the source sample is not biased by some selection criterium. More precisely, four (query) sources (L1551, L483, CB68, BHB07-11, and CB68) are characterized by $\mathrm{iCOM}$ emission, revealing hot corino activity. The next step is a careful investigation of weaker lines and the inspection of the images obtained using each array configuration, in order to provide a complete characterization of the molecular complexity in each source.

An example of what FAUST is able to provide when studying the inner 50 au around protostars is reported by Bianchi et al. (2020), who imaged the Class I L1551-IRS5 binary system in several species, namely, methanol (including ${ }^{13} \mathrm{CH}_{3} \mathrm{OH}$ and $\left.\mathrm{CH}_{2} \mathrm{DOH}\right)$, methyl formate $\left(\mathrm{HCOOCH}_{3}\right)$, and ethanol
$\left(\mathrm{CH}_{3} \mathrm{CH}_{2} \mathrm{OH}\right)$. Figure 3A shows the dust continuum $1.3 \mathrm{~mm}$ emission revealing the binary components, namely, the $\mathrm{N}$ (northern) and $\mathrm{S}$ (southern) objects. The two sources are surrounded by a circumbinary disk, clearly traced by CruzSáenz de Miera et al. (2019). The 50 au resolution reveals an iCOM-rich hot corino toward the $\mathrm{N}$ component (see the $\mathrm{HCOOCH}_{3}$ map in Figure 3B) and, in addition, a possible second hot corino around the $\mathrm{S}$ object. The number of hot corinos imaged around Class I sources is, so far, limited to a handful number (e.g., De Simone et al., 2017; Yang et al., 2021). The discovery of iCOM emission in L1551-IRS5 is, thus, instructive in the effort of tracking how the chemical richness around Class 0 objects is inherited by later Class I protostars: as a matter of fact, it looks that chemistry does not dramatically change. Projects on this topic based on statistically reliable samples will be hopefully performed soon. Figure 3B shows the map of the velocity peak of the $\mathrm{HCOOCH}_{3}\left(18_{4,14}-17_{4,13} \mathrm{~A}\right)$ line profile. A clear velocity gradient is revealed perpendicular to the axes of the protostellar jets (see the arrows in the Left panel) driven by $\mathrm{N}$ and $\mathrm{S}$. In conclusion, the chemical enrichment occurs in rotating hot corinos around the protostars and/or in the rotating circumbinary disk.

Moving to larger spatial scales (from 50 au to $1800 \mathrm{au}$ ), FAUST recently published a review of the molecular emission in the Class 0 IRAS15398-3359 star-forming region in Lupus (Okoda et al., 2021). The FAUST multi-species approach (CO, $\mathrm{C}^{18} \mathrm{O}$, SO, $\mathrm{H}_{2} \mathrm{CO}, \mathrm{CH}_{3} \mathrm{OH}$, and $\mathrm{SiO}$ ) allows Okoda et al. (2021) to well trace the dense and/or shocked material around the protostellar object. Besides the already known main outflow located along the NE-SW direction, a narrow $\left(\sim 1 \mathrm{~km} \mathrm{~s}^{-1}\right)$ line emission of shock tracers such as $\mathrm{SiO}$ and $\mathrm{CH}_{3} \mathrm{OH}$ reveals (i) a secondary outflow with an axis normal to the main flow, and (ii) an arc-like structure related with the secondary outflow (see the schematic picture in Figure 4). Which is the origin of the secondary outflow? Are the outflows driven by different protostars? As a matter of fact, there is no evidence (neither in the FAUST continuum maps) of a companion of the Class 0 IRAS15398-3359 protostar. An alternative solution proposed by Okoda et al. (2021) is that the 
secondary outflow is a relic of a past reorientation of the outflow launched from a single protostar and that the arc-like structure has dissipated the turbulence of the old shocked material. In principle, a dramatic change in the direction of the outflow axis could be possible if the molecular core hosting the protostar is associated with a nonuniform internal angular momentum, transferred onto the central region via episodic accretion.

Finally, the FAUST synergy is at work also to prepare complementary observations to those collected with the ALMA Large Program. More specifically, we started pilot projects in the $\mathrm{cm}$-spectral window using the very large array (VLA) interferometer. Given the dust opacity at $\mathrm{cm}$-wavelengths is negligible (e.g., Testi et al., 2014, and references therein), the main goal is to evaluate the effects of dust emission on Solar System scales, associated with both high volume and column densities (e.g., Miotello et al., 2014; Galván-Madrid et al., 2018; Galametz et al., 2019) on the collected iCOM images (and derived abundances) at (sub-) mm-wavelengths. An instructive example is provided by De Simone et al. (2020), who imaged at $1.3 \mathrm{~cm}$ at VLA in the FAUST context, the NGC1333-IRAS4A binary system. One of the two components 4A1 lacks iCOM emission when observed at millimeter wavelengths (see Figure 5), while the other component 4A2 is very rich in iCOMs. De Simone et al. (2020) found that, once imaged at $1.3 \mathrm{~cm}$, methanol lines are similarly bright toward $4 \mathrm{~A} 1$ and $4 \mathrm{~A} 2$, proving that both are hot corinos and not only 4A2. Complementing the ALMA observations with centimeter images can be then considered the future step which the FAUST synergy will perform. The VLA-ALMA combination will allow us to perform steps ahead in the study of the protostellar environments and to understand the ultimate molecular complexity in regions with a high column density of the material and consequently high dust opacity.

\section{DATA AVAILABILITY STATEMENT}

The original contributions presented in the study are included in the article/Supplementary Materials, further inquiries can be directed to the corresponding author.

\section{REFERENCES}

Aikawa, Y., and Herbst, E. (1999). Molecular Evolution in Protoplanetary Disks. Two-Dimensional Distributions and Column Densities of Gaseous Molecules. A\&A 351, 233-246.

André, P., Di Francesco, J., Ward-Thompson, D., Inutsuka, S.-I., Pudritz, R. E., and Pineda, J. (2014). From Filamentary Networks to Dense Cores in Molecular Clouds: Toward a New Paradigm for Star Formation. Protostars and Planets VI, 27-51. doi:10.2458/azu_uapress_9780816531240-ch002

Andrews, S. M., Huang, J., Pérez, L. M., Isella, A., Dullemond, C. P., Kurtovic, N. T., et al. (2018). The Disk Substructures at High Angular Resolution Project (DSHARP). I. Motivation, Sample, Calibration, and Overview. ApJ 869, L41. doi:10.3847/2041-8213/aaf741

Balbus, S. A., and Hawley, J. F. (1998). Instability, Turbulence, and Enhanced Transport in Accretion Disks. Rev. Mod. Phys. 70, 1-53. doi:10.1103/RevModPhys.70.1

Balucani, N., Ceccarelli, C., and Taquet, V. (2015). Formation of Complex Organic Molecules in Cold Objects: the Role of Gas-phase Reactions. MNRAS 449, L16-L20. doi:10.1093/mnrasl/slv009

\section{AUTHOR CONTRIBUTIONS}

All authors listed have made a substantial, direct, and intellectual contribution to the work and approved it for publication.

\section{FUNDING}

This project has received funding from: 1) the European Research Council (ERC) under the European Union's Horizon 2020 research and innovation program, for the Project The Dawn of Organic Chemistry (DOC), grant agreement No 741 002; 2) the PRIN-INAF 2016 The Cradle of Life-GENESIS-SKA (General Conditions in Early Planetary Systems for the rise of life with SKA); 3) a grant-in-aid from the Japan Society for the Promotion of Science (KAKENHI: Nos. 18H05222, 19H05069, 19K14753); 4) ANR of France under the contract number ANR-16-CE310013; 5) the French National Research Agency in the framework of the Investissements d'Avenir program (ANR-15-IDEX-02), through the funding of the Origin of Life project of the Univ. Grenoble-Alpes, 6) the European Union's Horizon 2020 research and innovation programs under projects Astro-Chemistry Origins (ACO), Grant No 811312.

\section{ACKNOWLEDGMENTS}

This manuscript is written on behalf of the whole FAUST team (http://faust-alma.riken.jp), and it makes use of the following ALMA data: ADS/JAO.ALMA\#2018.1.01205.L. ALMA is a partnership of ESO (representing its member states), NSF (United States), and NINS (Japan), together with NRC (Canada), MOST and ASIAA (Taiwan), and KASI (Republic of Korea), in cooperation with the Republic of Chile. The Joint ALMA Observatory is operated by ESO, AUI/ NRAO, and NAOJ. The National Radio Astronomy Observatory is a facility of the National Science Foundation operated under cooperative agreement by Associated Universities, Inc. This is a short text to acknowledge the contributions of specific colleagues, institutions, or agencies that aided the efforts of the authors.

Bianchi, E., Chandler, C. J., Ceccarelli, C., Codella, C., Sakai, N., López-Sepulcre, A., et al. (2020). FAUST I. The Hot Corino at the Heart of the Prototypical Class I Protostar L1551 IRS5. MNRAS 498, L87-L92. doi:10.1093/mnrasl/slaa130

Bisbas, T. G., Haworth, T. J., Barlow, M. J., Viti, S., Harries, T. J., Bell, T., et al. (2015). TORUS-3DPDR: a Self-Consistent Code Treating Three-Dimensional Photoionization and Photodissociation Regions. Mon. Not. R. Astron. Soc. 454, 2828-2843. doi:10.1093/mnras/stv2156

Caselli, P., and Ceccarelli, C. (2012). Our Astrochemical Heritage. Astron. Astrophys Rev. 20, 56. doi:10.1007/s00159-012-0056-x

Caselli, P., Vastel, C., Ceccarelli, C., van der Tak, F. F. S., Crapsi, A., and Bacmann, A. (2008). Survey of ortho-H\$ $\backslash$ mathsf $\left\{\_\{2\}\right\} \$ D \$$ mathsf $\{\{+\}\} \$(1 \$ \backslash$ mathsf \{ $\{1,0\}\}$ \$-1\$mathsf $\{\{1,1\}\} \$)$ in Dense Cloud Cores. A\&A 492, 703-718. doi:10.1051/0004-6361:20079009

Ceccarelli, C., Bacmann, A., Boogert, A., Caux, E., Dominik, C., Lefloch, B., et al. (2010). Herschelspectral Surveys of star-forming Regions. AઐA 521, L22. doi:10.1051/0004-6361/201015081

Ceccarelli, C., Caselli, P., Bockelée-Morvan, D., Mousis, O., Pizzarello, S., Robert, F., et al. (2014a). "Deuterium Fractionation: The Ariadne's Thread from the Precollapse Phase to Meteorites and Comets Today," in 
Protostars and Planets VI. Editors H. Beuther, R. S. Klessen, C. P. Dullemond, and T. Henning (Springer), 859. doi:10.2458/ azu_uapress_9780816531240-ch037

Ceccarelli, C., Caselli, P., Herbst, E., Tielens, A. G. G. M., and Caux, E. (2007). Extreme Deuteration and Hot Corinos: The Earliest Chemical Signatures of Low-Mass Star Formation. Protostars and Planets V, 47-62.

Ceccarelli, C., Dominik, C., López-Sepulcre, A., Kama, M., Padovani, M., Caux, E., et al. (2014b). Herschel Finds Evidence for Stellar Wind Particles in a Protostellar Envelope: Is This what Happened to the Young Sun? ApJ 790, L1. doi:10.1088/2041-8205/790/1/L1

Ceccarelli, C., Hollenbach, D. J., and Tielens, A. G. G. M. (1996). Far-Infrared Line Emission from Collapsing Protostellar Envelopes. ApJ 471, 400-426. doi:10.1086/177978

Ceccarelli, C., Viti, S., Balucani, N., and Taquet, V. (2018). The Evolution of Grain Mantles and Silicate Dust Growth at High Redshift. MNRAS 476, 1371-1383. doi:10.1093/mnras/sty313

Codella, C., Ceccarelli, C., Caselli, P., Balucani, N., Barone, V., Fontani, F., et al. (2017). Seeds of Life in Space (SOLIS). AઐA 605, L3. doi:10.1051/0004-6361/ 201731249

Codella, C., Ceccarelli, C., Lee, C.-F., Bianchi, E., Balucani, N., Podio, L., et al. (2019). The HH 212 Interstellar Laboratory: Astrochemistry as a Tool to Reveal Protostellar Disks on Solar System Scales Around a Rising Sun. ACS Earth Space Chem. 3, 2110-2121. doi:10.1021/ acsearthspacechem.9b00136

Cruz-Sáenz de Miera, F., Kóspál, Á., Ábrahám, P., Liu, H. B., and Takami, M. (2019). Resolved ALMA Continuum Image of the Circumbinary Ring and Circumstellar Disks in the L1551 IRS 5 System. ApJ 882, L4. doi:10.3847/2041$8213 /$ ab39ea

De Simone, M., Ceccarelli, C., Codella, C., Svoboda, B. E., Chandler, C., Bouvier, M., et al. (2020). Hot Corinos Chemical Diversity: Myth or Reality? ApJ 896, L3. doi:10.3847/2041-8213/ab8d41

De Simone, M., Codella, C., Testi, L., Belloche, A., Maury, A. J., Anderl, S., et al. (2017). Glycolaldehyde in Perseus Young Solar Analogs. AઐA 599, A121. doi:10.1051/0004-6361/201630049

Dulieu, F., Congiu, E., Noble, J., Baouche, S., Chaabouni, H., Moudens, A., et al. (2013). How Micron-Sized Dust Particles Determine the Chemistry of Our Universe. Sci. Rep. 3, 1338. doi:10.1038/srep01338

Fedele, D., Tazzari, M., Booth, R., Testi, L., Clarke, C. J., Pascucci, I., et al. (2018). ALMA Continuum Observations of the Protoplanetary Disk AS 209. A\&A 610, A24. doi:10.1051/0004-6361/201731978

Frank, A., Ray, T. P., Cabrit, S., Hartigan, P., Arce, H. G., Bacciotti, F., et al. (2014). Jets and Outflows from Star to Cloud: Observations Confront Theory. Protostars and Planets VI, 451-474. doi:10.2458/azu_uapress_9780816531240-ch020

Galametz, M., Maury, A. J., Valdivia, V., Testi, L., Belloche, A., and André, P. (2019). Low Dust Emissivities and Radial Variations in the Envelopes of Class 0 Protostars: Possible Signature of Early Grain Growth. A\&A 632, A5. doi:10.1051/0004-6361/201936342

Galván-Madrid, R., Liu, H. B., Izquierdo, A. F., Miotello, A., Zhao, B., CarrascoGonzález, C., et al. (2018). On the Effects of Self-Obscuration in the (Sub) Millimeter Spectral Indices and the Appearance of Protostellar Disks. ApJ 868, 39. doi:10.3847/1538-4357/aae779

Garrod, R. T., Weaver, S. L. W., and Herbst, E. (2008). Complex Chemistry in Starforming Regions: An Expanded Gas-Grain Warm-up Chemical Model. ApJ 682, 283-302. doi:10.1086/588035

Guillet, V., Pineau des Forêts, G., and Jones, A. P. (2011). Shocks in Dense Clouds. A\&A 527, A123. doi:10.1051/0004-6361/201015973

Herbst, E., and van Dishoeck, E. F. (2009). Complex Organic Interstellar Molecules. Annu. Rev. Astron. Astrophys. 47, 427-480. doi:10.1146/annurev-astro-082708101654

Higuchi, A. E., Sakai, N., Watanabe, Y., López-Sepulcre, A., Yoshida, K., Oya, Y., et al. (2018). Chemical Survey toward Young Stellar Objects in the Perseus Molecular Cloud Complex. ApJS 236, 52. doi:10.3847/1538-4365/ aabfe9
Ilee, J. D., Forgan, D. H., Evans, M. G., Hall, C., Booth, R., Clarke, C. J., et al. (2017). The Chemistry of Protoplanetary Fragments Formed via Gravitational Instabilities. MNRAS 472, 189-204. doi:10.1093/mnras/stx1966

Jørgensen, J. K., Belloche, A., and Garrod, R. T. (2020). Astrochemistry during the Formation of Stars. Annu. Rev. Astron. Astrophys. 58, 727-778. doi:10.1146/ annurev-astro-032620-021927

Lee, C.-F., Codella, C., Li, Z.-Y., and Liu, S.-Y. (2019). First Abundance Measurement of Organic Molecules in the Atmosphere of HH 212 Protostellar Disk. ApJ 876, 63. doi:10.3847/1538-4357/ab15db

Lee, C.-F., Ho, P. T. P., Li, Z.-Y., Hirano, N., Zhang, Q., and Shang, H. (2017a). A Rotating Protostellar Jet Launched from the Innermost Disk of HH 212. Nat. Astron. 1, 0152. doi:10.1038/s41550-017-0152

Lee, C.-F., Li, Z.-Y., Ho, P. T. P., Hirano, N., Zhang, Q., and Shang, H. (2017b). Formation and Atmosphere of Complex Organic Molecules of the HH 212 Protostellar Disk. ApJ 843, 27. doi:10.3847/1538-4357/aa7757

Lesaffre, P., Pineau des Forêts, G., Godard, B., Guillard, P., Boulanger, F., and Falgarone, E. (2013). Low-velocity Shocks: Signatures of Turbulent Dissipation in Diffuse Irradiated Gas. Aઐ $A$ 550, A106. doi:10.1051/0004-6361/201219928

Miotello, A., Testi, L., Lodato, G., Ricci, L., Rosotti, G., Brooks, K., et al. (2014). Grain Growth in the Envelopes and Disks of Class I Protostars. A $\varangle$ 567, A32. doi:10.1051/0004-6361/201322945

Okoda, Y., Oya, Y., Francis, L., Johnstone, D., Inutsuka, S.-i., Ceccarelli, C., et al. (2021). FAUST. II. Discovery of a Secondary Outflow in IRAS 15398-3359: Variability in Outflow Direction during the Earliest Stage of Star Formation? ApJ 910, 11. doi:10.3847/1538-4357/abddb1

Oya, Y., Sakai, N., López-Sepulcre, A., Watanabe, Y., Ceccarelli, C., Lefloch, B., et al. (2016). Infalling-Rotating Motion and Associated Chemical Change in the Envelope of IRAS 16293-2422 Source A Studied with ALMA. ApJ 824, 88. doi:10.3847/0004-637X/824/2/88

Podio, L., Garufi, A., Codella, C., Fedele, D., Bianchi, E., Bacciotti, F., et al. (2020). ALMA Chemical Survey of Disk-Outflow Sources in Taurus (ALMA-DOT). AઐA 642, L7. doi:10.1051/0004-6361/202038952

Sakai, N., Oya, Y., Higuchi, A. E., Aikawa, Y., Hanawa, T., Ceccarelli, C., et al. (2017). Vertical Structure of the Transition Zone from Infalling Rotating Envelope to Disk in the Class 0 Protostar, IRAS04368+2557. Mon. Not. R. Astron. Soc. Lett. 467, slx002-L80. doi:10.1093/mnrasl/slx002

Sakai, N., Oya, Y., Sakai, T., Watanabe, Y., Hirota, T., Ceccarelli, C., et al. (2014a). A Chemical View of Protostellar-Disk Formation in L1527. ApJ 791, L38. doi:10.1088/2041-8205/791/2/L38

Sakai, N., Sakai, T., Hirota, T., Watanabe, Y., Ceccarelli, C., Kahane, C., et al. (2014b). Change in the Chemical Composition of Infalling Gas Forming a Disk Around a Protostar. Nature 507, 78-80. doi:10.1038/nature13000

Sakai, N., and Yamamoto, S. (2013). Warm Carbon-Chain Chemistry. Chem. Rev. 113, 8981-9015. doi:10.1021/cr4001308

Sheehan, P. D., and Eisner, J. A. (2017). Disk Masses for Embedded Class I Protostars in the Taurus Molecular Cloud. ApJ 851, 45. doi:10.3847/1538-4357/aa9990

Skouteris, D., Vazart, F., Ceccarelli, C., Balucani, N., Puzzarini, C., and Barone, V. (2017). New Quantum Chemical Computations of Formamide Deuteration Support a Gas-phase Formation of This Prebiotic Molecule. Mon. Not. R. Astron. Soc. Lett. 468, slx012-L5. doi:10.1093/mnrasl/slx012

Stäuber, P., Doty, S. D., van Dishoeck, E. F., and Benz, A. O. (2005). X-ray Chemistry in the Envelopes Around Young Stellar Objects. A\&A 440, 949-966. doi:10.1051/0004-6361:20052889

Tabone, B., Cabrit, S., Bianchi, E., Ferreira, J., Pineau des Forêts, G., Codella, C., et al. (2017). ALMA Discovery of a Rotating SO/SO2 Flow in HH212. A $~ A 607$, L6. doi:10.1051/0004-6361/201731691

Testi, L., Birnstiel, T., Ricci, L., Andrews, S., Blum, J., Carpenter, J., et al. (2014). "Dust Evolution in Protoplanetary Disks," in Protostars and Planets VI. Editors H. Beuther, R. S. Klessen, C. P. Dullemond, and T. Henning (Springer), 339. doi:10.2458/azu_uapress_9780816531240-ch015

van 't Hoff, M. L. R., Harsono, D., Tobin, J. J., Bosman, A. D., van Dishoeck, E. F., Jørgensen, J. K., et al. (2020). Temperature Structures of Embedded Disks: Young Disks in Taurus Are Warm. ApJ 901, 166. doi:10.3847/1538-4357/abbla2 
Walsh, C., Nomura, H., and van Dishoeck, E. (2015). The Molecular Composition of the Planet-Forming Regions of Protoplanetary Disks across the Luminosity Regime. AઐA 582, A88. doi:10.1051/0004-6361/201526751

Watanabe, N., Kimura, Y., Kouchi, A., Chigai, T., Hama, T., and Pirronello, V. (2010). Direct Measurements of Hydrogen Atom Diffusion and the Spin Temperature of Nascent H 2 Molecule on Amorphous Solid Water. ApJ 714, L233-L237. doi:10.1088/2041-8205/714/2/L233

Wirström, E. S., and Charnley, S. B. (2018). Revised Models of Interstellar Nitrogen Isotopic Fractionation. MNRAS 474, 3720-3726. doi:10.1093/mnras/stx3030

Yang, Y.-L., Sakai, N., Zhang, Y., Murillo, N. M., Zhang, Z. E., Higuchi, A. E., et al. (2021). The Perseus ALMA Chemistry Survey (PEACHES). I. The Complex Organic Molecules in Perseus Embedded Protostars. ApJ 910, 20. doi:10.3847/ 1538-4357/abdfd6

Zhao, B., Caselli, P., Li, Z.-Y., Krasnopolsky, R., Shang, H., and Nakamura, F. (2016). Protostellar Disc Formation Enabled by Removal of Small Dust Grains. Mon. Not. R. Astron. Soc. 460, 2050-2076. doi:10.1093/mnras/stw1124
Conflict of Interest: The authors declare that the research was conducted in the absence of any commercial or financial relationships that could be construed as a potential conflict of interest.

Publisher's Note: All claims expressed in this article are solely those of the authors and do not necessarily represent those of their affiliated organizations, or those of the publisher, the editors, and the reviewers. Any product that may be evaluated in this article, or claim that may be made by its manufacturer, is not guaranteed or endorsed by the publisher.

Copyright (C) 2021 Codella, Ceccarelli, Chandler, Sakai, Yamamoto and The FAUST Team. This is an open-access article distributed under the terms of the Creative Commons Attribution License (CC BY). The use, distribution or reproduction in other forums is permitted, provided the original author(s) and the copyright owner(s) are credited and that the original publication in this journal is cited, in accordance with accepted academic practice. No use, distribution or reproduction is permitted which does not comply with these terms. 